\title{
Three-state lithography model: an enhanced mathematical approach to predict resist characteristics in grayscale lithography processes
}

\author{
Bassem Badawi $\odot$,* Olfa Sayadi $\odot$, Ignaz Eisele, and Christoph Kutter \\ Fraunhofer EMFT Research Institution for Microsystems and Solid State Technologies, \\ Department of Silicon Technologies and Devices, Munich, Germany
}

\begin{abstract}
Background: Physical modeling of grayscale lithography processes for the prediction of photoresist heights leads to complex mathematical algorithms. A promiment example is the numerical simulation of the photoresist shape after development through Dill's equations. These grayscale lithography models exhibit accurate prediction quality but can not directly implemented into mask layout tools to simplify the layout procedure. Limited process windows, changes in the mask design, variations of the used materials or manufacturing tools lead to time-consuming and cost-intensive test procedures to adjust the photoresist model for sufficient results.
\end{abstract}

Aim: The focus of this work is to enhance current grayscale lithography models for a straightforward method with the same precise prediction of remaining photoresist heights to simplify the mask layout process. Moreover, we aim for an uncomplicated optimization of the model to minimize the empirical analysis necessary for its use.

Approach: Based on experimental results, we deploy a sectionally defined mathematical expression that includes the theory of Fraunhofer diffraction and illumination-dependent activation of the photo-sensitive component and its solubility in developer.

Results: We produced pyramidal, spherical and chess field structures with exposure doses of 3000 and $15,000 \mathrm{~J} / \mathrm{m}^{2}$ on bare silicon substrates with $100-\mathrm{nm}$ resolution and on silicon substrates with anti-reflective coatings, with accuracy as fine as $20 \mathrm{~nm}$.

Conclusion: The proposed three-state lithography model has been verified by experimental evaluation. It is able to operate in a wide process window and can be directly implemented in existing mask layout software. This model ensures a cost-efficient and precisely controlled production of three-dimensional topographies using grayscale lithography processes.

(C) The Authors. Published by SPIE under a Creative Commons Attribution 4.0 Unported License. Distribution or reproduction of this work in whole or in part requires full attribution of the original publication, including its DOI. [DOI: 10.1117/1.JMM.20.1.014601]

Keywords: grayscale; gray-tone; lithography; photoresist; modeling; topography.

Paper 20023 received May 25, 2020; accepted for publication Nov. 23, 2020; published online Jan. 15, 2021.

\section{Introduction}

Over the past three decades, grayscale lithography has steadily gained interest both in industrial and scientific community. The ability to create desired three-dimensional (3D) topographies on a surface through a cost-efficient method enabled grayscale lithography to improve the field of micro-optics. ${ }^{1,2}$ The semiconductor industry exploits the advantages of grayscale lithography for the generation of special ion-implantation profiles, e.g., for the junction termination extension technique. ${ }^{3}$ Investigations by Schneider et al. ${ }^{4}$ revealed the possibilities of sharp 3D-ion implantation profiles on several simulations using semiconductor applications. However, recent research leverages grayscale lithography for production of blazed gratings in miniaturized

*Address all correspondence to Bassem Badawi, bassem.badawi@emft.fraunhofer.de 
spectrometers, solid emitters for electrospray ionization applications, and liquid lenses for use in optogenetics. ${ }^{5}$

The generation of arbitrary grayscales can be accomplished through various lithography technologies, i.e., e-beam, x-ray, or traditional UV exposure. This work utilizes UV exposure for structuring of photo-sensitive resists, as it is a common technique in the semiconductor industry. Due to complex mask preparation methods and their demanding manufacturing requirements, this technology becomes very time-consuming and cost-intensive for the production of 3D topographies. To achieve varying lateral height levels in a resist, the exposure dose must be modified laterally. The current resist technologies yield high contrasts, resulting in optimal imaging properties. This requires precise control of the energy exposure directed at the photosensitive resist. An effective principle to control the exposure intensity was published by Waits et al. ${ }^{6}$ and utilizes the theory of Fraunhofer diffraction via sub-resolution patterns on a photomask. Prior to this, the mathematical background for this approach was presented in 1995 by Wagner et al. ${ }^{7}$ As reported by Smith et al., ${ }^{5}$ the usage of current simulation tools for grayscale lithography processes can lead to limited process windows in which only specific mask features for certain exposures yield acceptable results. Their work showed process windows for mask features with an areal density of 0.45 and 0.35 at SPR518 and SPR220 photoresist with possible exposure doses between 2000 and $3000 \mathrm{~J} / \mathrm{m}^{2}$ and 2200 and $4000 \mathrm{~J} / \mathrm{m}^{2}$.

Standard lithography simulation tools apply Dill's equations, ${ }^{8}$ which use the numerical solution of time-dependent differential equations to simulate the inhibitor concentration in thin, vertically stacked photoresist layers for lateral exposure intensity, calculated using the Fourier transformation of the mask and a given modulation transfer function. An empirically acquired development rate for specific inhibitor concentrations is used in conjunction with Dill's equations to calculate the resist solubility. The approach in this work simplifies this current mathematical process to simulate the photoresist solubility with an effortless method, which is accessible in mask layout software tools. Moreover, this mathematical concept enables an easy optimization procedure improving upon current simulation applications. This model has been given the name "three-state lithography model" due to the three different solubility phases that occur in the photoresist during exposure.

\section{Materials and Method}

\subsection{Experimental}

For the experimental procedure, we utilize a postive AZ ECI 3027 photoresist for all test processes and the pyramidal, spherical, and chess field sample structures. The target shape designs of the pyramidal, chess field, and spherical structures are shown in Figs. 1-3. Operating on a Suess Gamma spin coating and developing system, resist thicknesses of $3 \mu \mathrm{m}$ were coated on 200-mm silicon substrates. The UV exposures were performed using a Canon FPA-3000 i4 Stepper with an i-line $(\lambda=365 \mathrm{~nm})$ illumination system, a numerical aperture $N A=0.62$, and an object to image magnification ratio $M=5: 1$. No post-exposure bakes were executed before developing in AZ 726 MIF. To flatten the resist irregularities on the pyramidal resist structures, a subsequent

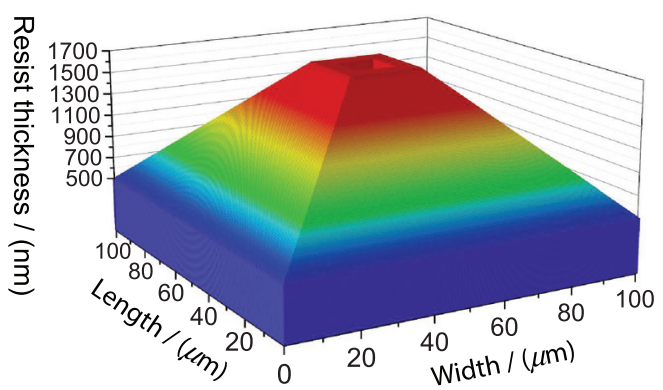

Fig. 1 A 3D layout of the pyramid topography with a base length of $100 \mu \mathrm{m}$ and a slope angle of $\approx 2.4^{\circ} \mu \mathrm{m}$. The tip of the pyramid is replaced by a quadratic cavity with a surface area of $11 \mu \mathrm{m} \times$ $11 \mu \mathrm{m}$ and a depth of $1,1 \mu \mathrm{m}$. 


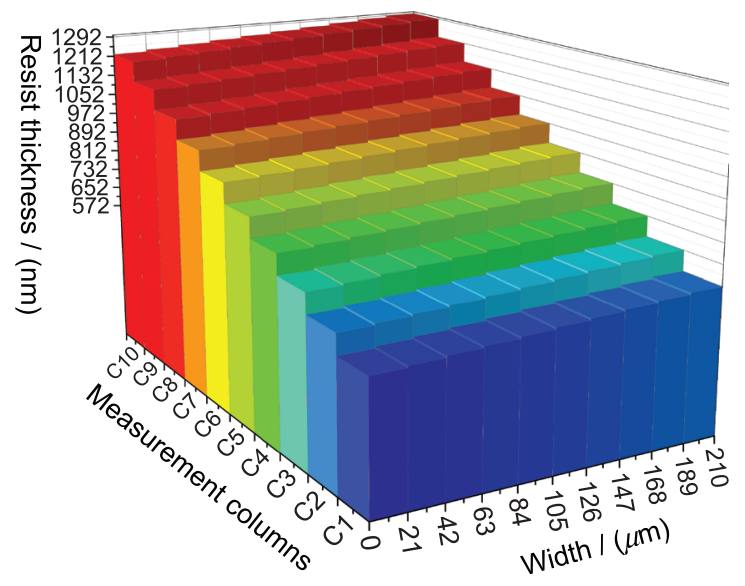

Fig. 2 A 3D layout of the chess field topography with a base length of $210 \mu \mathrm{m}$ and height variation of $8 \mathrm{~nm}$ in a range of 500 to $1292 \mathrm{~nm}$ between the $21 \mu \mathrm{m} \times 21 \mu \mathrm{m}$ fields.

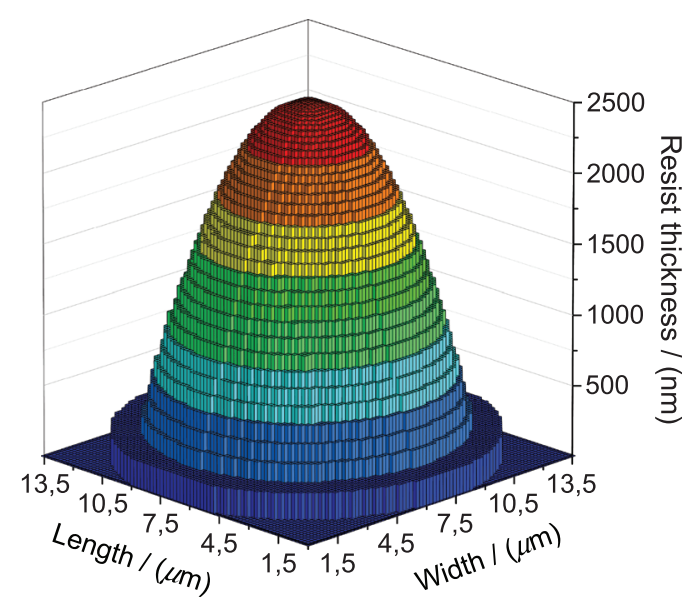

Fig. 3 A 3D layout of the spherical topography with a base diameter of $12.3 \mu \mathrm{m}$ and a height of $2.5 \mu \mathrm{m}$.

hard-bake at $115^{\circ} \mathrm{C}$ was performed for $120 \mathrm{~s}$. Thickness measurements of the contrast curve were carried out using a KLA-Tencor UV1280. The resultant pyramidal and chess field structures were investigated by KLA Tencor P-17 Stylus Profiler, while the spherical structures are inspected using a Digital Instruments Dimension 5000 Atomic Force Microscope (AFM) system.

To handle the high amount of data necessary for mask design, a combination of MATLAB and Tanner L-Edit scripts were used for the automatic generation of mask features and their positioning on the mask based on the three-state photoresist model. For this purpose, a test reticle was produced containing squared fields, with side lengths of $2.7 \mathrm{~mm}$, consisting of quadratic shapes with a pitch of $1.5 \mu \mathrm{m}$ and fill factors from 0.04 to 1 . For sufficient validation of the process window of the proposed model, similar test layouts were fabricated using exposure doses of 3000 and $15,000 \mathrm{~J} / \mathrm{m}^{2}$. For additional verification of the model, all sample structures were created in both lightfield and darkfield ambients to prove the development uniformity under different exposure conditions.

\subsection{Modeling}

Reliable height predictions require precise knowledge of the contrast function for a given resist and developer combination. Moreover, this function or contrast curve includes specific process 
parameters, such as the optical absorption and reflection properties of the substrate, and as applied soft-bake and developing process parameters. To compensate for the asymmetric course of the contrast curve, the exposure dose of the measured heights were corrected under consideration of the resist's inner light absorption by the Lambert-Beer law using the boundary condition of a constant absorption coefficient $\alpha$ during exposure. Subsequently, the corrected data set was fitted to a modified sigmoid function (henceforth designated s-function), which matches best to the contrast curve data of our measurments and directly correlates to the production of the soluble photoresist component (carboxylic acid) during exposure. This correction determines a threshold energy dose needed to start the chemical transformation of the photo-sensitive component.

As a result, the mathematical expression for the measured contrast curve can be written as

$$
T=\frac{D_{0}}{1+e^{\left(a E e^{-\alpha\left(D_{0}-T\right)}+b\right)}},
$$

where $a$ and $b$ are the parameters extracted from the corrected data, whereas $D_{0}$ represents the initial resist thickness.

As presented in the work of Wagner et al., ${ }^{7}$ rectangular sub-resolution mask patterns with a width $x$ and length $y$ separated through a constant pitch $p$ can be applied to alter the intensity distribution in the areal image. Out of these parameters, a fill factor GS for mask features is expressed as

$$
\mathrm{GS}=\frac{x y}{p^{2}}
$$

Considering the Rayleigh resolution limit, we have set $p$ to $300 \mathrm{~nm}$ for the used i-line projection system. The physical basis of intensity variations for specific mask features is a limited entrance pupil of the projection system, which acts as an optical low-pass filter. The diffraction image plane in a Köhler illumination system is located in the entrance pupil position. Considering ray optics, the object-sided opening angle $\theta$ can be given as

$$
\theta=\frac{2 \mathrm{NA}}{M},
$$

where $N A$ is the numerical aperture and $M$ represents the magnification ratio. The mathematical treatment of the intensity distribution is derived from the theory of Fraunhofer diffraction ${ }^{10}$ and describes the propagation of an incident plane wave on a lattice in the far field. For a twodimensional quadratic aperture, the intensity $I_{d}$ of the diffraction pattern is proportional to

$$
I_{d} \propto(x y)^{2}\left(\frac{\sin \left(x \frac{\pi}{\lambda} \sin (\alpha)\right)}{x \frac{\pi}{\lambda} \sin (\alpha)}\right)^{2}\left(\frac{\sin \left(y \frac{\pi}{\lambda} \sin (\beta)\right)}{y \frac{\pi}{\lambda} \sin (\beta)}\right)^{2},
$$

where $\alpha$ and $\beta$ represent the angular divergence of the diffracted light in $x$ and $y$ direction and $\lambda$ is the illumination wavelength. For a squared-entrance pupil geometry, the two-dimensional integral of Eq. (4) over the angles of divergence determines the intensity in the aerial image plane and is expressed as

$$
I_{i} \propto \frac{2}{\pi}(x y)^{2} \int_{0}^{\theta / 2} \int_{0}^{\theta / 2} I_{d}(\alpha, \beta) \mathrm{d} \alpha \mathrm{d} \beta,
$$

where $\theta / 2$ acts as spatial cut-off frequency of the optical low-pass filter. The impact of altering intensities on the contrast curve in Eq. (1) can be taken into account by $k_{F}$ (henceforth designated Fourier factor), which includes the contribution of accessible intensity propagating through the entrance pupil. The Fourier factor is expressed using Eq. (2) as

$$
k_{F}=\mathrm{GS}^{2}\left(\frac{2 k_{x} \operatorname{Si}\left(2 k_{x}\right)+\cos \left(2 k_{x}\right)-1}{\pi k_{x}}\right)\left(\frac{2 k_{y} \operatorname{Si}\left(2 k_{y}\right)+\cos \left(2 k_{y}\right)-1}{\pi k_{y}}\right),
$$




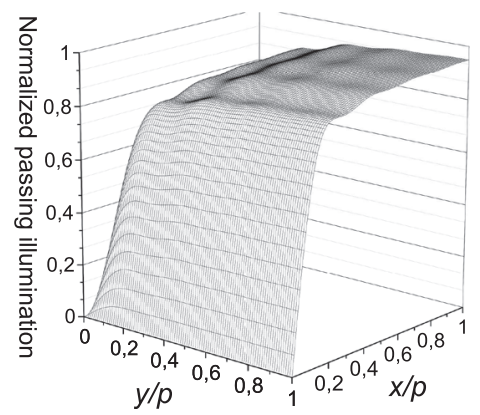

Fig. 4 Contribution of diffraction to $k_{F}$ in dependence on the standardized mask pattern dimensions $x / p$ and $y / p$.

where the expression $\operatorname{Si}()$ represents the sine integral, $k_{x}=\pi x \sin (\alpha) / \lambda$ and $k_{y}=\pi y \sin (\beta) / \lambda$. Through multiplication of Eq. (6) with the exposure dose $E$ in Eq. (1), the contrast curve is described by the expression

$$
T=\frac{D_{0}}{1+e^{\left(a k_{F} E e^{-\alpha\left(D_{0}-T\right)}+b\right)}} .
$$

A closer examination on Eq. (6) reveals that the transmitted light is not merely proportional to the squared fill factor, but also dependent on the transmitted wavelength, system entrance pupil, and the pattern size. The part of illumination passing the entrance pupil of the optical system applied in this work is shown in Fig. 4 and is dependent on the size of the mask features. To ensure the Fourier factor is dependent exclusively on the fill factor, mask features are limited to quadratic shapes. This limitation provides the additional benefit of lowering the calculation efforts needed for the model. In this case, $k_{F}$ becomes one-dimensional. The course of $k_{F}$ for quadratic mask features compared to a simple quadratic dependency is shown in Fig. 5.

As a consequence of lower intensities for narrower mask patterns, the threshold for chemical transformation in the resist shifts to higher energy doses. This shifted threshold $E_{\text {shift }}$ is dependent on $G S$ and is added to the initial threshold energy dose $E$ in Eq. (1) with respect to the expression

$$
E_{\text {shift }}=-c \ln (\mathrm{GS}) e^{-d \mathrm{GS}} \text {, }
$$

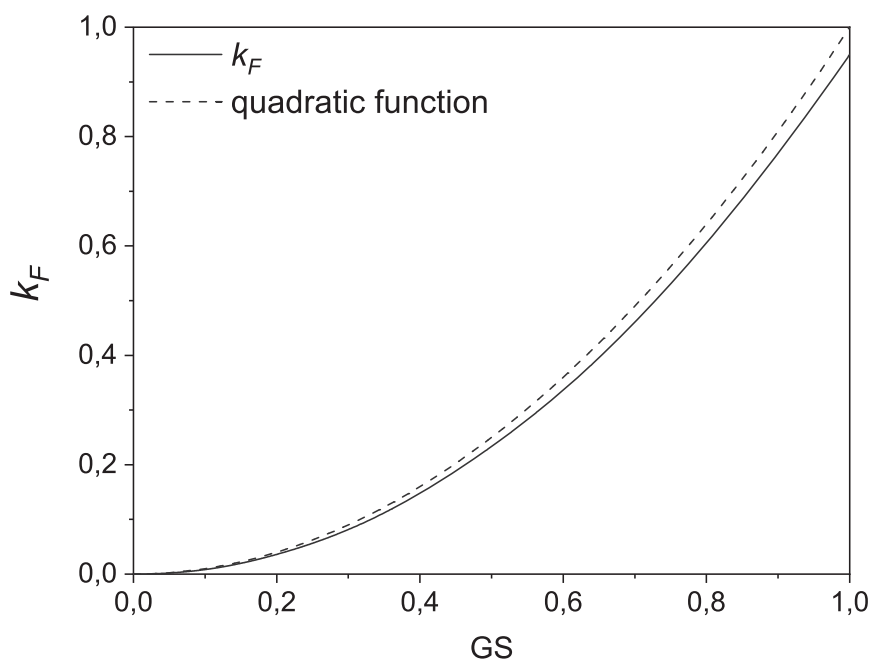

Fig. 5 Fourier factor $k_{F}$ for quadratic mask pattern compared to the quadratic function $k_{F}=\mathrm{GS}^{2}$. 
where $c$ and $d$ represent characteristic parameters for each type of photoresist. These parameters are empirically determined by exposing a test mask to a variable exposure dose.

During exposure, the optical properties of the positive photoresist change due to the chemical formation of carboxylic acid. The chemical transformation, which primarily impacts the extinction coefficient, causes the photoresist to appear transparent to UV radiation. This effect, known as bleaching, is strongly dependent on the chemical compounds of the photoresist and affects the slope of the contrast curve individually for any mask pattern fill factor. To a good approximation, the soluble photoresist component near the resist-developer surface is modeled by utilizing Eqs. (1) and (8) without taking Lambert-Beer into account. The solubility of deeperlying material within the photoresist follows Eq. (7). To create a continuous transition between the shallow and deep photoresist solubilities, an intermediate phase is mathematically expressed through a spline-interpolation function and is defined individually, dependent on the fill factor. Therefore, the three-state photoresist model follows the sectionally defined expression

$$
E= \begin{cases}\frac{\ln \left(D_{0} / T-1\right)+b}{a}-E_{\text {shift }} & T>P_{1} \\ e T^{3}+f T^{2}+g T+h & T<P_{1} \wedge T>P_{2} \\ \frac{\ln \left(D_{0} / T-1\right)+b}{a k_{F} e^{-\alpha\left(D_{0}-T\right)}} & T \leq P_{2}\end{cases}
$$

where the boundary points $P_{1}$ and $P_{2}$ represent the intersections between the phases. The symbols $e, f, g$, and $h$ operate as fit parameters for the spline function. To decrease calculation complexity, the implicit expressions in Eqs. (1) and (7) are expressed in their explicit forms in Eq. (9), dependent on the photoresist thickness $T$.

\section{Verification and Discussion}

In this work, a dose range from 600 to $3000 \mathrm{~J} / \mathrm{m}^{2}$ discloses the contrast curve shape. The measured resist thickness $T$ dependent on the exposure dose $E$, the data after the correction and the s-functions are shown in Fig. 6 for photoresist on bare silicon and on a bottom antireflective coating $(\mathrm{BARC}) /$ silicon surface.

Contrast curve calculation examples of different fill factors are shown in Fig. 7. To a first approximation, the boundary point $P_{1}$ is set to $2950 \mathrm{~nm}$ and $P_{2}$ is set to Eq. (9a) $\cap$ Eq. (9c) $-2 \cdot\left(P_{1}-\right.$ Eq. (9a) $\cap$ Eq. (9c)). The intermediate phase depth of the individual contrast curves shows a linear dependence on the fill factor. For a fill factor of $\approx 0.40$,

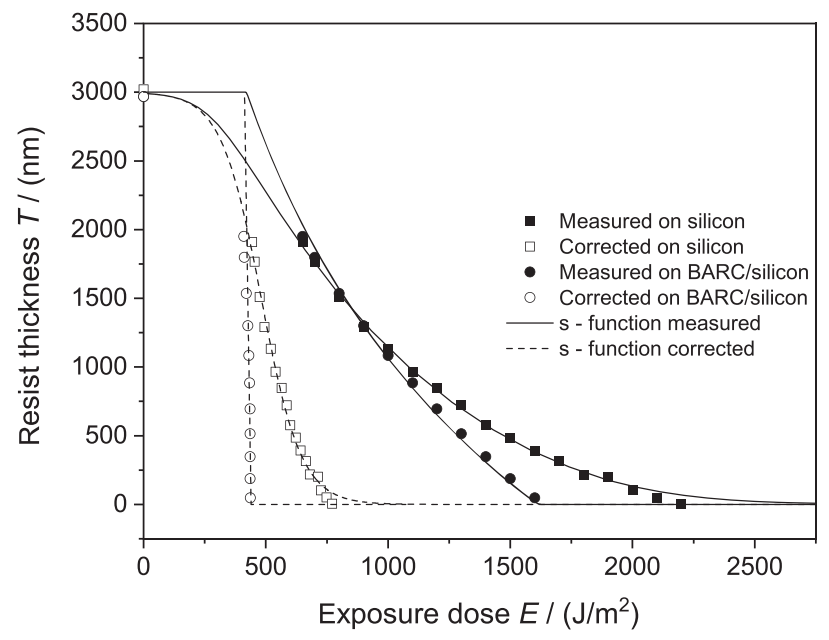

Fig. 6 Measured and corrected resist heights and s-functions after dose range from 600 to $3000 \mathrm{~J} / \mathrm{m}^{2}$ for photoresist on bare silicon and on a BARC/silicon surface. The correction procedure is explained in detail in Sec. 2.2. 


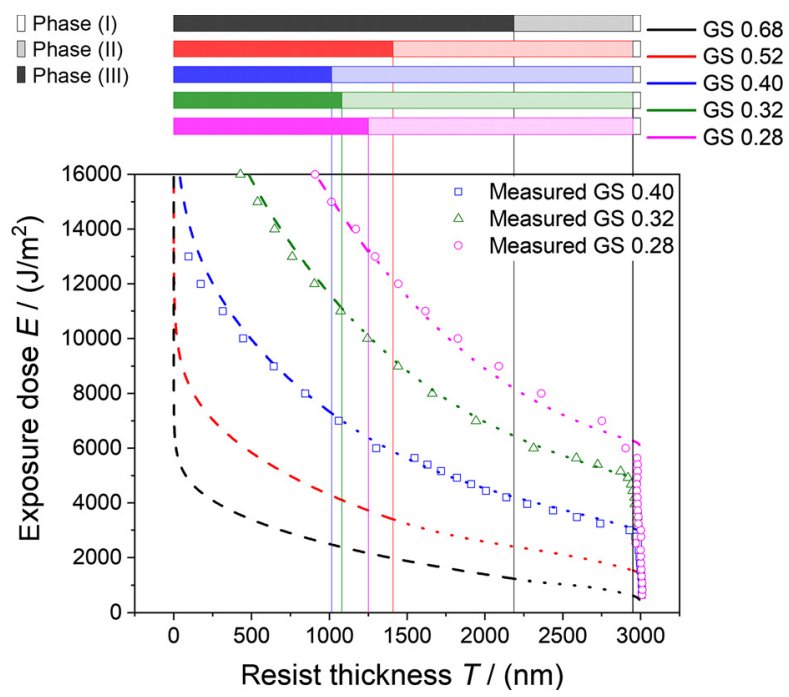

Fig. 7 Contrast curves for photoresist on bare silicon for the fill factors $G S=0.28,0.32,0.40$, 0.52 , and 0.68 calculated by the three-state resist model in comparison to sample measurements of the resist thickness produced with a test mask. Straight lines corresponding to Eq. (9a), dotted lines corresponding to Eq. (9b), and dashed lines to Eq. (9c).

the intermediate phase reaches its maximum depth, before the intermediate phase shrinks for higher fill factors. This physical phenomenon is called reciprocity failure, and was investigated in detail by Sheats ${ }^{11}$ in 1988. Accordingly, the chemical meta-stable product absorbance during the carboxylic acid production is shown to be responsible for this effect. To validate the simulated contrast curves, Fig. 7 shows measurement data produced with the test mask out of Sec. 2.1 for fill factors $\mathrm{GS}=0.28,0.32$, and 0.40 , which follow the simulated contrast curve courses. Accordingly, the model enables the accurate prediction of remaining resist heights in a wide range of low to high exposure doses.

Figure 8 shows two instances of the photoresist pyramidal structure at different positions (site I and site II) on a wafer compared to the target design topography. Furthermore, Fig. 8 also illustrates the topography after a hard-bake procedure for each energy dose and in both lightfield and darkfield ambients.

The formation of a standing wave within the photoresist results in a minimum height step of $\approx 100 \mathrm{~nm}$, which corresponds to the constructive interference locations within the standing wave, as seen on the faces of the pyramidal structures. The topographies show discrepancies between the designed and resultant topographies of up to $200 \mathrm{~nm}$. In darkfield ambient, both exposure doses yield similar profiles. Thickness discrepancies between lightfield and darkfield ambients

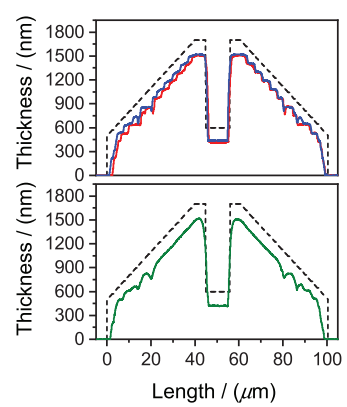

(a)

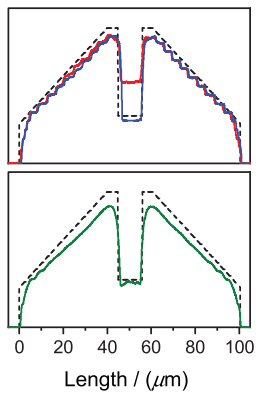

(b)

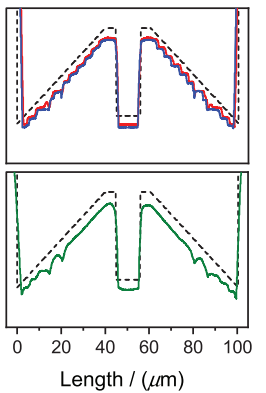

(c)

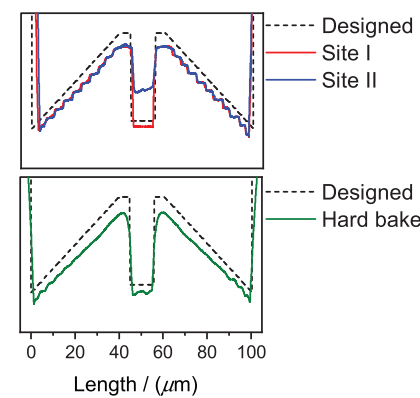

(d)

Fig. 8 Resulting pyramidal photoresist topographies before and after hard-bake in lightfield ambients with exposure doses of (a) 15,000 and (b) $3000 \mathrm{~J} / \mathrm{m}^{2}$ and darkfield ambients with exposure doses of (c) 15,000 and (d) $3000 \mathrm{~J} / \mathrm{m}^{2}$. 


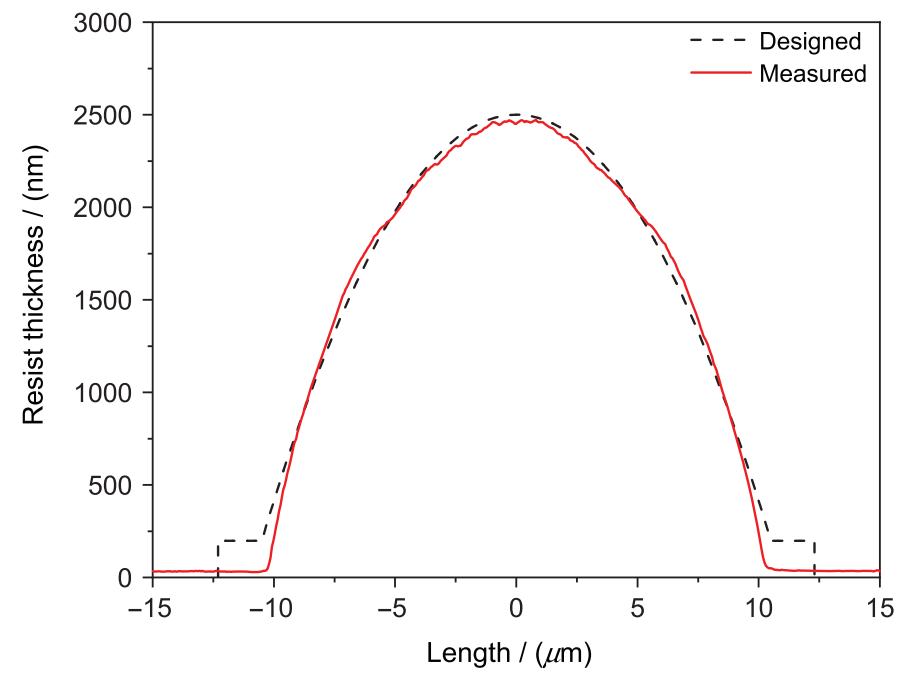

Fig. 9 Measured height profile of the sphere structure on BARC/silicon surface and a exposure of $3000 \mathrm{~J} / \mathrm{m}^{2}$ compared to the target design in Fig. 3.

of 40 to $60 \mathrm{~nm}$ were observed for $15,000 \mathrm{~J} / \mathrm{m}^{2}$, while the lower dose of $3000 \mathrm{~J} / \mathrm{m}^{2}$ led to variations of 100 to $200 \mathrm{~nm}$. The higher instability at lower exposure doses against ambient is caused by the decreased exposure time, resulting in less irregularly formed carboxylic acid. Additionally, this effect led to better roughness properties for higher exposure doses. At an exposure dose of $3000 \mathrm{~J} / \mathrm{m}^{2}$, the produced pyramid slope exhibited inaccurate angles and sensitivity to ambient conditions. Conversely, an exposure dose of $15,000 \mathrm{~J} / \mathrm{m}^{2}$ yielded more desirable results. In particular, the cavities produced at $3000 \mathrm{~J} / \mathrm{m}^{2}$ showed inconsistent depths. The added hard-bake process led to smooth surfaces of the pyramidal structure. Due to a missing height step at a photoresist thickness of $\approx 700 \mathrm{~nm}$, the topographies at exposure doses of 15,000 J/m² still show irregularities.

To achieve a smooth surface without hard-bake post processing the silicon surface is coated with a BARC to avoid the formation of a standing wave within the photoresist. Due to this substrate modification, the contrast curve function changes, as shown in Fig. 6. To limit errors from the mask design for a contrast curve on bare silicon, the target sphere structure in Fig. 3 is fabricated only with an exposure dose of $3000 \mathrm{~J} / \mathrm{m}^{2}$. An AFM line measurement over the sphere center is shown in Fig. 9. Figure 10 shows a $30 \mu \mathrm{m} \times 30 \mu \mathrm{m}$ map over the sphere.

Although the mask design is not based on the substrate surface characteristics, the resultant resist sphere shows a satisfactory level of consistency compared to the target sphere design. The base of the sphere at $\approx 200 \mathrm{~nm}$ could not be printed into the resist due to the significantly steeper contrast curve course for thinner photoresist heights with BARC coating.

The manufactured chess field structures are shown in Figs. 11(a)-11(c). Here the simulated step designs for BARC/silicon sufaces are adjusted in consideration of the changed contrast curve in Fig. 6.

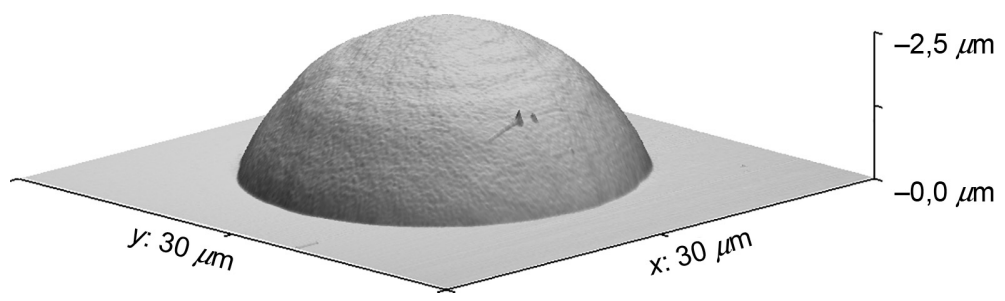

Fig. 10 Measured 3D topography of the sphere structure with BARC/silicon surface and a exposure of $3000 \mathrm{~J} / \mathrm{m}^{2}$ 


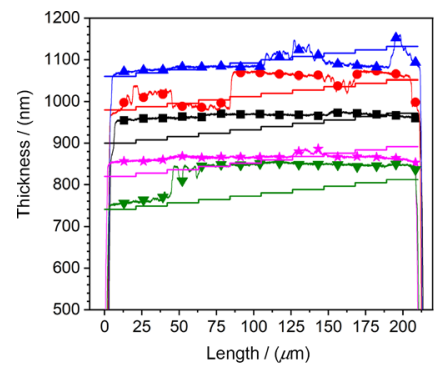

(a)

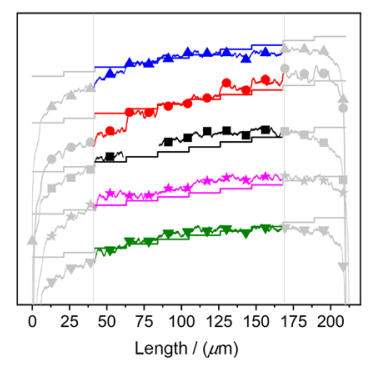

(b)

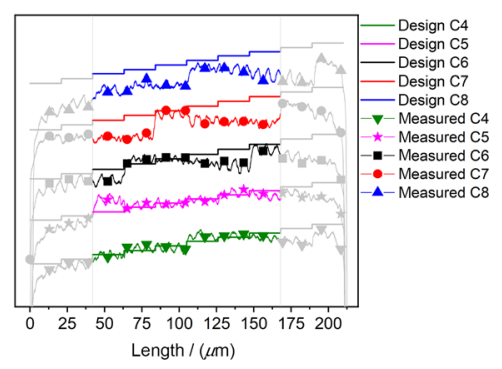

(c)

Fig. 11 Resulting example step topographies of the target chess field in Fig. 2 for an exposure of $3000 \mathrm{~J} / \mathrm{m}^{2}$ in (a) on bare silicon substrate and on BARC/silicon substrate for an exposure of $15000 \mathrm{~J} / \mathrm{m}^{2}$ in (b) and $3000 \mathrm{~J} / \mathrm{m}^{2}$ in (c). First two steps in (b) and (c) on structure edges are neglected.

As seen previously, height variation under $100 \mathrm{~nm}$ are not resolvable on a bare silicon substrate [Fig. 11(a)] due to standing wave effects within the photoresist. The BARC/silicon substrate optimization reveals a vertical resolution limit of $\approx(20$ to $40 \mathrm{~nm})$ for $15,000 \mathrm{~J} / \mathrm{m}^{2}$ [Fig. 11(b)], if structure edges are neglected. This leads to a continuous height variation and not into a stepwise vertical change of $\sim 8 \mathrm{~nm}$ between the fields. The continuous slope is caused by the chemical resolution limit of the photoresist, which is usually applied for lateral critical dimensions around $350 \mathrm{~nm}$. Moreover, higher exposure intensities [Fig. 11(c)] create rougher surfaces, which further increases the resolution limit.

\section{Conclusion}

The mathematical procedure proposed in this paper describes the change of the soluble photoresist component dependent on mask features and fixed illumination settings for the production of arbitrary 3D topographies. The capabilities of the model were demonstrated through the creation of pyramidal, spherical and chess field photoresist structures with a prediction accuracy down to 20 to $40 \mathrm{~nm}$. An additional parameter optimization process using the three-state lithography model will lead to an increasing prediction quality. The mathematical approach was applied in a wide process window between 3000 and $15,000 \mathrm{~J} / \mathrm{m}^{2}$, dependent on given optical system parameters and mask features with fill factors between 0.17 and 0.81 . Process variations or changes in fabrication tools and materials are easily accounted for in the procedure and do not require large empirical tests to examine the change of the development rate for specific inhibitor concentrations, as presented by Dill et al. ${ }^{8}$ Moreover, this straightforward method enables an adequate prediction of remaining photoresist after development without numerical techniques used in commercial simulation software, such as PROLITH, ${ }^{12}$ to solve time dependent differential equations. The simplified mathematical description of the photoresist solubility allows for easy integration within standard mask layout tools. Thus, the mask layout for grayscale applications is feasible without additional simulation tools. The three-state lithography model enables a cost-efficient method for the production of 3D topographical structures in micro-electromechanical systems (MEMS), micro-opto-electro-mechanical systems (MOEMS) and semiconductor applications with a high degree of accuracy. Due to substantial simplifications in the mathematical description of the photoresist and light physics, the proposed model is unable to simulate diffusion processes or interference effects. Therefore, the three-state lithography model is targeted for the use in grayscale lithography applications and is not developed to replace the available, sophisticated numerical simulation tools.

\section{Acknowledgments}

Bassem Badawi, Olfa Sayadi, Professor Dr. Ignaz Eisele, and Professor Dr. Christoph Kutter declare that they have no conflict of interest. 


\section{References}

1. B. Morgan et al., "Development of a deep silicon phase fresnel lens using gray-scale lithography and deep reactive ion etching," J. Microelectromech. Syst. 13(1), 113-120 (2004).

2. J. Xiao et al., "Fabrication of CMOS-compatible optical filter arrays using gray-scale lithography," J. Micromech. Microeng. 22(2), 025006 (2012).

3. E. A. Imhoff, F. J. Kub, and K. D. Hobart, "Grayscale junction termination for high-voltage sic devices," Mater. Sci. Forum 615-617, 691-694 (2009).

4. J. Schneider et al., "3D lithography for implant applications," in Int. Semiconductor Conf. Dresden-Grenoble, IEEE, pp. 1-4 (2013).

5. M. A. Smith et al., "Design, simulation, and fabrication of three-dimensional microsystem components using grayscale photolithography," J. Micro/Nanolithogr. MEMS MOEMS 18(4), 043507 (2019).

6. C. M. Waits, A. Modafe, and R. Ghodssi, "Investigation of gray-scale technology for large area 3D silicon MEMS structures," J. Micromech. Microeng. 13(2), 170-177 (2003).

7. B. Wagner et al., "Microfabrication of complex surface topographies using grey-tone lithography," Sens. Actuators A 46(1-3), 89-94 (1995).

8. F. H. Dill et al., "Modeling projection printing of positive photoresists," IEEE Trans. Electron Devices 22(7), 456-464 (1975).

9. C. Mack, Fundamental Principles of Optical Lithography: The Science of Microfabrication, Reprinted with Corr ed., Wiley, Chichester (2012).

10. B. E. A. Saleh and M. C. Teich, Fundamentals of Photonics, Wiley Series in Pure and Applied Optics, 2nd ed., John Wiley \& Sons, Chichester (2013).

11. J. R. Sheats, "Reciprocity failure in novolak/diazoquinone photoresist with 364-nm exposure," IEEE Trans. Electron Devices 35(1), 129-131 (1988).

12. C. A. Mack, "Inside Prolith TM: a comprehensive guide to optical lithography simulation: for the Prolith family of lithography simulation tools," v5.0, Finle Technologies, Austin, Texas (1997).

Bassem Badawi is a PhD student at the Munich University of the Federal Armed Forces and a scientific researcher at the Fraunhofer Research Institution for Microsystems and Solid State Technologies EMFT in Munich, Germany. He received his BS and MS degrees in engineering physics and micro- and nanotechnology from the University of Applied Sciences Munich in 2013 and 2017, respectively. His current research interests include lithography technology, $\gamma$-radiation sensors, MEMS, and MOEMS applications.

Olfa Sayadi was a student from the Technical University of Munich and contributed in this work for the purpose of her master's study completion. She graduated with a Master of Science (MSc).

Ignaz Eisele was appointed professor at the University of the Federal Armed Forces in Munich in 1979 and held the professorship for Microsystems Technology at the Faculty for Electrical Engineering and Information Technology. He was honored as the first Excellent Emeritus of the University of the Federal Armed Forces in Munich in 2009 and has been leading the Fraunhofer EMFT Department of Silicon Technologies and Devices (SiD).

Christoph Kutter has headed the Fraunhofer Institute for Microsystems and Solid State Technologies EMFT since July 2012. With a research focus on sensor and actuator solutions. He holds the professorship in the field of solid-state technologies at the University of the Federal Armed Forces in Munich. From 1995 to 2012, he held various management positions at Infineon Technologies AG and Siemens AG. 